\title{
Rapid occurrence of left ventricular thrombus associated with platinum-based chemotherapy plus cetuximab for the treatment of metastatic squamous cell carcinoma of the head and neck: A case report
}

\author{
ATSUSHI IKEDA ${ }^{1}$, EIKI YAMACHIKA ${ }^{1}$, MASAHIDE MIZUTANI ${ }^{2}$, MASAKAZU MATSUBARA $^{1}$, \\ NORIFUMI MORITANI ${ }^{1}$, KAZUKI NAKATSUJI ${ }^{1}$ and SEIJI IIDA ${ }^{1}$ \\ ${ }^{1}$ Department of Oral and Maxillofacial Reconstructive Surgery, Okayama University Hospital, Okayama, Okayama 700-8558; \\ ${ }^{2}$ Department of Dentistry, Oral and Maxillofacial Surgery, Osaka Police Hospital, Osaka, Osaka 543-0035, Japan
}

Received December 8, 2016; Accepted June 2, 2017

DOI: $10.3892 /$ mco.2017.1393

\begin{abstract}
Platinum-based chemotherapy plus cetuximab represents the first-line treatment for recurrent or metastatic squamous cell carcinoma of the head and neck. The most common adverse events associated with cetuximab are infusion reactions and skin reactions, and a risk of venous thromboembolic events has also recently been reported in association with cetuximab. It is well known that thrombosis is a common complication of malignancy, and represents the second most frequent cause of mortality in cancer patients. The present study reports the case of a 79-year-old man who presented with lung and liver metastases from tongue squamous cell carcinoma, for which platinum-based chemotherapy plus cetuximab was administered. After 1 cycle, the patient showed rapid growth of a left ventricular (LV) thrombus, despite ongoing antiplatelet therapy for an old myocardial infarction. Anticoagulant therapy was administered to treat the LV thrombus, which resolved within 1 week. To the best of our knowledge, this represents the first reported case of rapidly occurring LV thrombus associated with platinum-based chemotherapy plus cetuximab. Platinum-based chemotherapy
\end{abstract}

Correspondence to: Dr Atsushi Ikeda, Department of Oral and Maxillofacial Reconstructive Surgery, Okayama University Hospital, 2-5-1 Shikata-cho, Kita-ku, Okayama, Okayama 700-8558, Japan

E-mail: i.atsushi@cc.okayama-u.ac.jp; ikeda-familia@lake.ocn.ne.jp

Abbreviations: SCC, squamous cell carcinoma; 5-FU, 5-fluorouracil; VTE, venous thromboembolic event; LV, left ventricular; FDG, fluorodeoxyglucose; PET, positron emission tomography; CT, computed tomography; EF, ejection fraction; DVT, deep vein thrombosis; TEE, venous and arterial thromboembolic event

Key words: cetuximab, head and neck, left ventricular thrombus, squamous cell carcinoma plus cetuximab may be associated with a higher risk of embolic thrombus.

\section{Introduction}

Platinum-based chemotherapy plus cetuximab represents the first-line treatment for recurrent or metastatic squamous cell carcinoma (SCC) of the head and neck (1). In the European Phase III EXTREME trial of patients with recurrent or metastatic SCC of the head and neck, the addition of cetuximab to platinum/5-fluorouracil (5-FU) in the first-line setting significantly improved the rates of overall survival, progression-free survival and overall response compared with platinum/5-FU alone (1). However, cetuximab treatment is associated with certain adverse events, the most common of which are infusion reactions and skin reactions. Additionally, a risk of venous thromboembolic events (VTEs) has recently been reported in association with cetuximab (1). It has been suggested that the association between cetuximab and risk of thrombosis may be due to the antiangiogenic effects of cetuximab (2).

VTEs are considered to be under-diagnosed, as patients are typically asymptomatic at presentation and are often only diagnosed incidentally (2). A previous study reported that the majority of VTEs $(92 \%)$ were identified during radiological examinations scheduled for tumor reevaluation (3).

The present study reports the case of a 79-year-old man who presented with lung and liver metastases from a tongue SCC, and suffered rapid growth of a left ventricular (LV) thrombus following 1 cycle of platinum-based chemotherapy plus cetuximab. Anticoagulant therapy was administered to treat the LV thrombus, which resolved within 1 week. The current report highlights the potential association between platinum-based chemotherapy plus cetuximab and the increased risk of embolic thrombus.

\section{Case report}

A 77-year old male patient initially presented to Department of Oral and Maxillofacial Reconstructive Surgery, Okayama 
University Hospital (Okayama, Japan) in October 2012 with a chief complaint of a painless tumor in the left tongue accompanied by ulceration. The tumor was elastic and hard, and measured $13 \times 11 \mathrm{~mm}$. Tongue SCC (cT1N0M0) was diagnosed from a biopsy specimen. The patient's history included an old myocardial infarction, for which he was undergoing treatment with antiplatelet drugs (acetylsalicylic acid, $100 \mathrm{mg} /$ day; ticlopidine hydrochloride, $100 \mathrm{mg} /$ day). Glossectomy was performed in November 2012.

However, 8 months later, left submandibular lymph node metastasis was detected. A left radical neck dissection was performed in May 2013, followed by adjuvant radiotherapy (total dose, $63 \mathrm{~Gy}$ ).

After a further 18 months, fluorodeoxyglucose (FDG)-positron emission tomography (PET) examination revealed lung and liver metastases. Computed tomography (CT) scans performed at the same time showed no LV thrombus (Fig. 1) and no reduction in ejection fraction [EF; 57\% (normal range, $>55 \%$ )]. Platinum-based chemotherapy plus cetuximab was initiated to treat the metastases. Due to the patient's age and decreased renal function, the regimen consisted of carboplatin (area under the curve, $5 \mathrm{mg} / \mathrm{ml} / \mathrm{min}$; 1-h intravenous infusion on day 1), 5 -FU (dose, $1,000 \mathrm{mg} / \mathrm{m}^{2} /$ day for 4 days), and cetuximab (initial dose, $400 \mathrm{mg} / \mathrm{m}^{2}$; followed by subsequent weekly doses of $250 \mathrm{mg} / \mathrm{m}^{2}$ ). After 1 cycle, CT revealed a ball-like, movable LV thrombus, measuring $13 \times 10 \mathrm{~mm}$ (Fig. 2). The patient had a good general status, normal results on electrocardiography, no reduction in EF (58\%), no chest pain, and no dyspnea. Laboratory data revealed a D-dimer level of $6.1 \mu \mathrm{g} / \mathrm{ml}$, a platelet count of $86 \times 10^{3} / \mu 1$, and a level of fibrin degradation products of $9.5 \mu \mathrm{g} / \mathrm{ml}$. The cause of the $\mathrm{LV}$ thrombus was unclear. To treat the LV thrombus, anticoagulant therapy (heparin, 14,000 U/day) was initiated. One day later, acute arterial thrombosis was detected in the radial artery. However, the LV and radial artery thrombi had completely disappeared after 1 week. The LV thrombus showed no recurrence.

Chemotherapy was then restarted and continued for a total of 6 cycles. Subsequent FDG-PET and CT scans revealed a reduction in the size of lung metastases; however, the size and number of liver metastases were increased, and the development of bone metastases was detected. The patient eventually succumbed to respiratory failure 6 months after commencing chemotherapy.

Informed consent for participation and for the publication of the results of present study was obtained from the patient.

\section{Discussion}

Cetuximab treatment is most commonly associated with infusion reactions and skin reactions, and an increased risk of VTEs has also been reported recently $(1,2)$. However, due to the typical lack of symptoms with VTEs, they are often only diagnosed incidentally $(2,3)$. In the present case, CT scans that were performed to evaluate the effectiveness of chemotherapy incidentally revealed LV thrombus. For detecting deep vein thrombosis (DVT), D-dimer has been reported to be extremely an useful marker (4), offering $84 \%$ sensitivity, $96 \%$ specificity, and $90 \%$ accuracy when using a cutoff value of $\geq 3.0 \mu \mathrm{g} / \mathrm{ml}$ (4). In the present case, the D-dimer level was $6.1 \mu \mathrm{g} / \mathrm{ml}$; DVT was not detected, but LV thrombus was revealed.

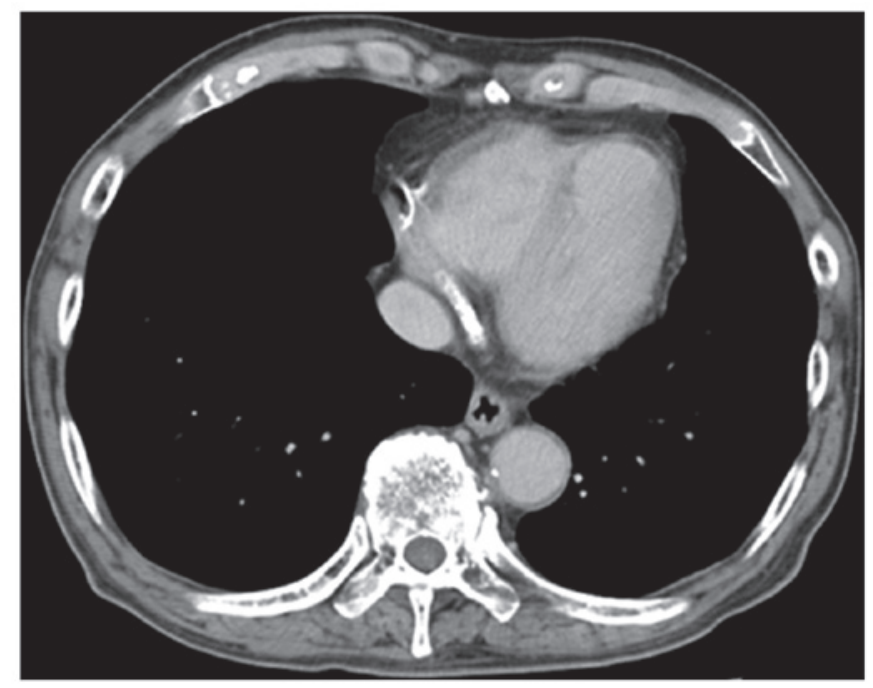

Figure 1. Computed tomography findings prior to chemotherapy. No thrombi were detected in the left ventricle.

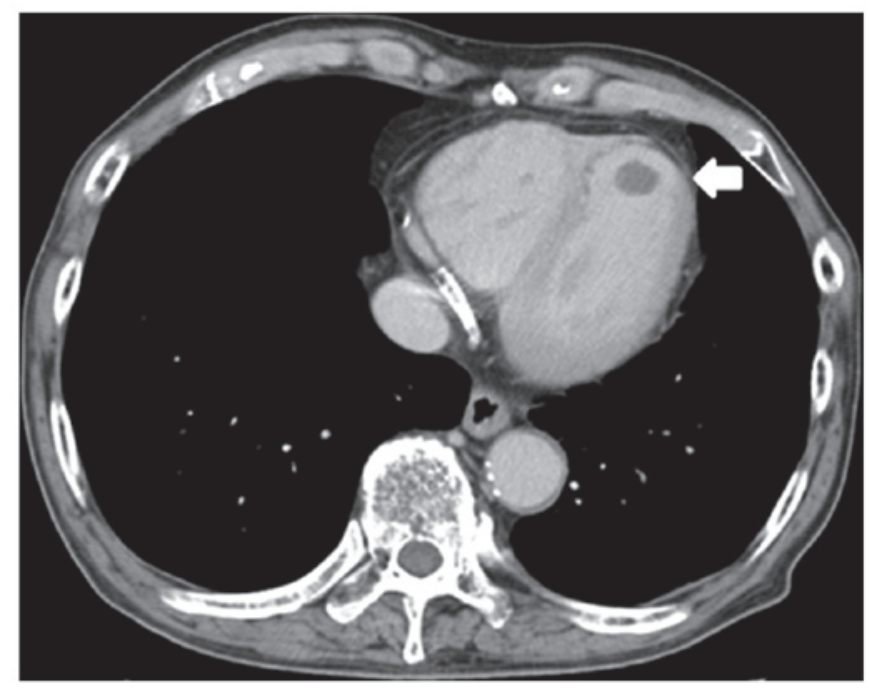

Figure 2. Computed tomography findings subsequent to chemotherapy. A left ventricular thrombus (arrow) measuring 13×10 mm was detected.

Transthoracic echocardiography is the initial imaging technique $(5,6)$ performed for detecting and diagnosing cardiac masses (5). It is able to reliably indicate the anatomical and functional features of a detected cardiac mass; however, it has a limited ability to characterize tissue. In this respect, cardiac magnetic resonance imaging is superior at present, and should thus be the standard diagnostic tool for the assessment of cardiac masses (5). It is easy to diagnose an LV thrombus if any clinical and diagnostic findings are present, including subjective symptoms, wall motion abnormalities, and changes on electrocardiography. Currently, routine VTE prophylaxis during chemotherapy in outpatient settings is not recommended by international guidelines; however, certain studies have suggested that the risk of VTE can be decreased by prophylactic low-molecular-weight heparin in patients with metastatic or locally advanced cancer who are receiving chemotherapy (7). 
A large retrospective analysis reported that cisplatin-based chemotherapy carried a high risk of venous and arterial thromboembolic events (TEEs), with $88 \%$ of TEEs occurring within 100 days of commencing cisplatin (8). TEEs included DVT alone in $49.7 \%$ of cases, pulmonary embolism alone in $25.4 \%$, DVT plus pulmonary embolism in $13.6 \%$, arterial TEEs alone in $8.3 \%$, and DVT plus arterial TEEs in $3.0 \%$ of cases. However, arterial TEEs did not include LV thrombus. In the same observation period, a mortality rate of $3.3 \%$ was noted, and it was considered that there was a strong probability of TEEs contributing to or causing the majority of these mortalities (8). Additionally, the incidence rate of TEE was higher in cases of metastatic disease $(21.6 \%)$ than in cases of early-stage $(16.7 \%)$ or locally advanced disease (15.2\%) (8). Cisplatin-associated vascular toxicities may include hypomagnesemia, increased levels of von Willebrand factor, and damage to endothelial cells via increased formation of procoagulant endothelial microparticles (9), and it is likely that their pathogenesis involves a pathway that affects arterial and venous systems (8). Although it has been suggested that the risk of VTEs increases when a patient is treated with combined cetuximab and platinum-based chemotherapy (2), no reports have precisely described what this risk level is. In a previous study, patients with malignancy showed a 4.1-fold increased risk of VTEs compared with those without malignancy, and the addition of chemotherapy increased that risk to 6.5-fold (10). Cardiac events, which represent a special category of adverse events, include five conditions: Arrest, arrhythmia, congestive heart failure, ischemia or infarction, and sudden death. In a previous study (1), in patients receiving platinum/5-FU plus cetuximab or platinum/5-FU alone, the predominant grade 3-4 cardiac events were congestive heart failure (4 patients and 1 patient, respectively), infarction and ischemia ( 7 and 2 patients, respectively), and sudden death (3 patients and 1 patient, respectively). In that study (1), the reason for sudden death was not clear; one possibility was the occurrence of an LV thrombus or a suspended thromboembolism.

Although LV thrombus is an uncommon primary disease, it is a common complication following acute myocardial infarction, and is associated with a risk of systemic embolism $(6,11)$. A movable LV thrombus is more likely to be associated with embolization compared with an immovable LV thrombus (11). Similarly, a thrombus that protrudes into the LV cavity (ball-like thrombus) is more likely to be associated with embolization compared with a flat thrombus (mural-type thrombus) $(5,11)$.

The treatment of LV thrombi remains controversial. Surgery is recommended if the general condition of the patient is sufficient and the thrombus is markedly protruding or is a floating-type thrombus. As fibrinolytic therapy may cause fresh LV thrombi to become fragmented and subsequently form emboli, anticoagulant therapy is used frequently (6). In the current case, anticoagulation therapy was selected. Acute arterial thrombosis of the radial artery occurred 1 day after the commencement of anticoagulant therapy; however, the LV and radial artery thrombi had completely disappeared after 1 week.

It has been reported that the ability of tumor cells to activate the coagulation system can lead to a hypercoagulable or prothrombotic state in cases of malignancy (12). This hypercoagulable state is associated with interactions between different mechanisms related to the activation of various hemostatic components, such as the coagulation and fibrinolytic pathways, vascular endothelium, monocytes, and platelets (12). Furthermore, functional DNA polymorphisms in genes encoding thrombosis-related factors have been associated with increased risk of oral SCC, and a number of single-nucleotide polymorphisms associated with thrombosis may serve as primary predictors for oral SCC risk (13).

A previous case report described the occurrence of an LV thrombus in a patient who had a medical history of cutaneous T-cell lymphoma and hypereosinophilia as well as a recent Mycoplasma pneumoniae infection (5). The authors hypothesized that there was an association between the Mycoplasma pneumoniae infection and the occurrence of arterial and venous thrombi. However, the underlying pathogenesis remained unclear, and the thrombus formation may have resulted from a hypercoagulable state induced by one or more of the patient's underlying diseases.

In the present case, no LV thrombus was apparent prior to chemotherapy. Despite the patient taking an antiplatelet agent, an LV thrombus suddenly arose within the first cycle of chemotherapy. The patient had no subjective symptoms, cardiac hypofunction, or acute myocardial infarction, and the LV thrombus disappeared rapidly. Neoplastic thrombi are often reported $(5,10,14)$. In the present case, it is possible that LV thrombus was caused by a neoplastic thrombus; however, the LV thrombus occurred rapidly following the initiation of chemotherapy. Additionally, although LV thrombi are rare and are most often caused by acute myocardial infarction $(6,11,15)$, a reduction of cardiac function was not detected in the present case. Thus, the reason for the occurrence of the LV thrombus was not clear. We hypothesize that the LV thrombus may be attributed to both the presence of a neoplastic thrombus and chemotherapy.

In the present case, the LV thrombus was identified early and was successfully cured. If chemotherapy had continued, serious thromboembolism may have occurred. The risks of thromboembolic events associated with cetuximab (2) and cisplatin-based chemotherapy (8) have been reported previously; however, it is possible that platinum-based chemotherapy plus cetuximab, as in the present case, may carry a higher risk of embolic thrombosis compared with cetuximab or platinum-based agents administered individually.

\section{References}

1. Vermorken JB, Mesia R, Rivera F, Remenar E, Kawecki A, Rottey S, Erfan J, Zabolotnyy D, Kienzer HR, Cupissol D, et al: Platinum-based chemotherapy plus cetuximab in head and neck cancer. N Engl J Med 359: 1116-1127, 2008.

2. Petrelli F, Cabiddu M, Borgonovo K and Barni S: Risk of venous and arterial thromboembolic events associated with anti-EGFR agents: A meta-analysis of randomized clinical trials. Ann Oncol 23: 1672-1679, 2012.

3. Mandala M, Barni S, Floriani I, Isa L, Fornarini G, Marangolo M, Mosconi S, Corsi D, Rulli E, Frontini L, et al: Incidence and clinical implications of venous thromboembolism in advanced colorectal cancer patients: The 'GISCAD-alternating schedule' study findings. Eur J Cancer 45: 65-73, 2009.

4. Sassa H, Sone T, Tsuboi H, Kondo J and Yabashi T: Diagnostic significance of thrombin-antithrombin III complex (TAT) and D-dimer in patients with deep venous thrombosis. Jpn Circ J 60: 201-206, 1996. 
5. Oeser C, Andreas M, Rath C, Habertheuer A and Kocher A Left ventricular thrombus in a patient with cutaneous T-cell lymphoma, hypereosinophilia and Mycoplasma pneumoniae infection-a challenging diagnosis: A case report. J Cardiothorac Surg 10: 21, 2015 .

6. Iga K, Kondo H, Tamura T, Izumi C, Inoko M, Kitaguchi S, Hirozane T, Himura Y, Gen H and Konishi T: Clinical characteristics of patients with fresh left ventricular thrombus. Jpn Circ J 64: 254-256, 2000

7. Agnelli G, Gussoni G, Bianchini C, Verso M, Mandalà M Cavanna L, Barni S, Labianca R, Buzzi F, Scambia G, et al: Nadroparin for the prevention of thromboembolic events in ambulatory patients with metastatic or locally advanced solid cancer receiving chemotherapy: A randomised, placebo-controlled, double-blind study. Lancet Oncol 10: 943-949, 2009.

8. Moore RA, Adel N, Riedel E, Bhutani M, Feldman DR, Tabbara NE, Soff G, Parameswaran R and Hassoun H: High incidence of thromboembolic events in patients treated with cisplatin-based chemotherapy: A large retrospective analysis. J Clin Oncol 29: 3466-3473, 2011.

9. Lechner D, Kollars M, Gleiss A, Kyrle PA and Weltermann A: Chemotherapy-induced thrombin generation via procoagulant endothelial microparticles is independent of tissue factor activity. J Thromb Haemost 5: 2445-2452, 2007.

10. Heit JA, Silverstein MD, Mohr DN, Petterson TM, O'Fallon WM and Melton LJ III: Risk factors for deep vein thrombosis and pulmonary embolism: A population-based case-control study. Arch Intern Med 160: 809-815, 2000
11. Haugland JM, Asinger RW, Mikell FL, Elsperger J and Hodges M: Embolic potential of left ventricular thrombi detected by two-dimensional echocardiography. Circulation 70: 588-598, 1984.

12. Caine GJ, Stonelake PS, Lip GY and Kehoe ST: The hypercoagulable state of malignancy: Pathogenesis and current debate Neoplasia 4: 465-473, 2002.

13. Vylliotis A, Yapijakis C, Nkenke E, Nisyrios T, Avgoustidis D, Adamopoulou M, Ragos V, Vassiliou S, Koronellos N and Vairaktaris E: Effect of thrombosis-related gene polymorphisms upon oral cancer: A regression analysis. Anticancer Res 33: 4033-4039, 2013.

14. Cogo A, Bernardi E, Prandoni P, Girolami B, Noventa F, Simioni P and Girolami A: Acquired risk factors for deep-vein thrombosis in symptomatic outpatients. Arch Intern Med 154: 164-168, 1994.

15. Asinger RW, Mikell FL, Elsperger J and Hodges M: Incidence of left-ventricular thrombosis after acute transmural myocardial infarction. Serial evaluation by two-dimensional echocardiography. N Engl J Med 305: 297-302, 1981. 